\title{
Muon production and accumulation from positrons on target
}

\author{
M. Boscolo $\odot,{ }^{1, *}$ M. Antonelli, ${ }^{1}$ A. Ciarma $\odot,{ }^{1}$ and P. Raimondi ${ }^{2}$ \\ ${ }^{1}$ INFN-LNF, Via E. Fermi 40, 00044 Frascati, Rome, Italy \\ ${ }^{2}$ ESRF, 71 avenue des Martyrs, 38000 Grenoble, France
}

(Received 30 January 2020; accepted 15 April 2020; published 8 May 2020; corrected 24 September 2020)

\begin{abstract}
In this paper we investigate the production of a muon beam by positrons on target. We describe the characteristics of the muon beam as produced by different target configurations. We present an optics for the muon accumulator ring, discussing how it fulfils the large energy acceptance requirement and the high order chromaticity correction at the target, as well as other parameters relevant to enhance the muon bunch quality. We discuss the muon beam dynamics through the ring for the configuration of the single-pass LEMMA scheme, and the optimization study performed with the goals of maximizing the muon bunch population and minimizing its emittance.
\end{abstract}

DOI: 10.1103/PhysRevAccelBeams.23.051001

\section{INTRODUCTION}

A novel approach has been recently proposed to produce low emittance muon beams using electron-positron collisions at a center-of-mass energy just above the $\mu^{+} \mu^{-}$ production threshold, corresponding to the direct annihilation of approximately $45 \mathrm{GeV}$ positrons and atomic electrons in a target. Previous studies of the Low EMittance Muon Accelerator (LEMMA) proposal are reported in Refs. [1-3]. A review of the proton-driven and positron-driven proposals can be found in Ref. [4].

The cross section for continuum muon pair production $e^{+} e^{-} \rightarrow \mu^{+} \mu^{-}$has a maximum value of about $1 \mu \mathrm{b}$ at $\sqrt{s} \sim 0.230 \mathrm{GeV}$. In our proposal these values of $\sqrt{s}$ can be obtained from fixed target interactions with a positron beam energy of about $45 \mathrm{GeV}$.

The conceptual scheme considered for the present studies, the single-pass scheme proposed in [1] and described in [3], is shown in Fig. 1. A preliminary analysis of the multipass scheme is reported in [2]. The single-pass scheme is now subject of deeper investigation as it provides a larger number of muons per bunch, an easier handling of thermomechanical issues, and a duty cycle compatible with the current acceleration schemes. However a sizeable emittance increase due to the use of a thicker target is expected. With respect to the scheme described in Refs. [1,3] we considered the possibility to use multiple extraction lines and recombine the bunches in the transverse plane, in a revolver configuration. Similar schemes

\footnotetext{
*manuela.boscolo@lnf.infn.it
}

Published by the American Physical Society under the terms of the Creative Commons Attribution 4.0 International license. Further distribution of this work must maintain attribution to the author(s) and the published article's title, journal citation, and DOI. have been designed to enhance the muon beam intensity for neutrino factories [5-7].

We present here the design of the muon accumulator ring with the target insertion region, and the muon beam dynamics through this ring. To optimize the muon pair production different elements play a role, mainly: the impinging positron beam energy, spot size and divergence, the target material and thickness. Previous studies on muon accumulation are described in [8].

In order to study the interplay of the different effects a simulation tool, MUFASA (MUon FAst Simulation Algorithm), has been developed. It is a $\mathrm{C}++$ based Monte Carlo code. It includes the relevant processes of muon and electron interactions with matter and particle tracking by using PTC interfaced with MAD-X [9]. The use of a fast simulation allows for a wide range of parametric studies and a detailed 6D beam dynamics analysis.

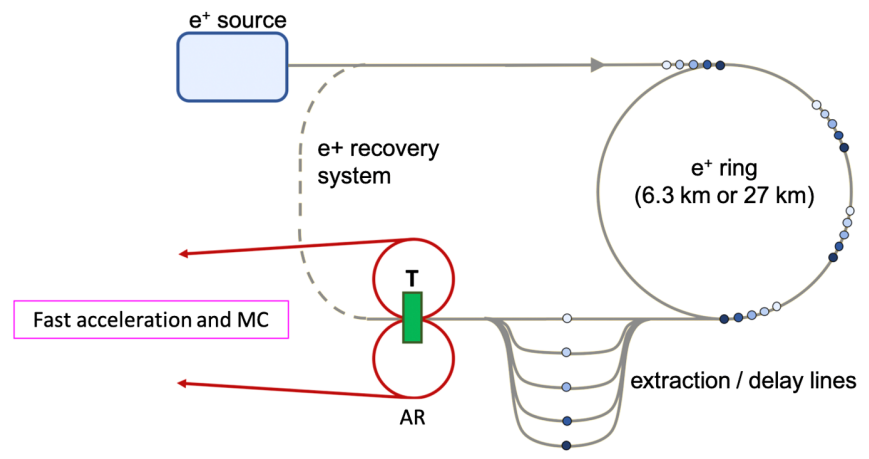

FIG. 1. Schematic layout for the muon beam production from positrons on target: $e^{+}$source, $e^{+}$ring, extraction lines and single target $(\mathrm{T})$ for $\mu^{+} \mu^{-}$production, two muon accumulator rings (AR), and fast acceleration section to be followed by the muon collider. 
The paper is organized as follows. In Sec. II we describe the accumulator ring optics with its most relevant characteristics and requirements for our needs. A novel high momentum acceptance accumulator ring with a proper insertion region has been designed in order to deal with the energy spread at production and the low cross section. In Sec. III we discuss different target options with the muon beam dynamics simulation studies. We conclude in Sec. IV by summarizing the results from the particle tracking simulation studies and presenting an outlook on the future studies.

\section{ACCUMULATOR RING DESIGN}

Muon pairs are produced by positrons hitting a target placed in a low- $\beta$ insertion region. The muons have an energy of about $22.5 \mathrm{GeV}$ and a lifetime of about $0.5 \mathrm{~ms}$. The muons are accumulated in separate rings, according to their charges; they cross the target at each turn, as schematically shown in Fig. 1. The optimal number of turns in the rings is found by particle tracking simulations and it depends on various parameters that will be discussed in the following.

The two key requirements that acted as drivers for the design of these two compact accumulator rings were the maximisation of the number of muons per bunch and the preservation of the production emittance.

Different versions of the lattice have been tested with particle tracking simulations, as well as various targets tested for the same optics. Results of these studies are discussed in the next Sec. III. We describe in this section the lattice version having the best performance in terms of muon production and accumulation.

The definitely novel optics design is able to provide very large momentum acceptance and small emittance. Low- $\beta$ is easily included in this lattice. These characteristics are essential for the preservation of the emittance and maximization of the number of particles in the muon bunch.

The lattice consists of two symmetrical arcs, each one composed of six cells and two straight sections, one for the target insertion and one for the radio-frequency (rf) section. The circumference is $140 \mathrm{~m}$ with dipole fields of $15 \mathrm{~T}$.

Figure 2 shows the corresponding optical functions for half ring, from the center of the insertion region to the center of the rf section. The four central cells are identical with each other, while the two exterior ones slightly differ to match with the incoming straight sections.

Figure 3 shows a central cell. One half cell is composed of three focusing and two defocusing quadrupoles, with two focusing and two defocusing sextupoles placed at the center of each quadrupole, and is about $8 \mathrm{~m}$ long. The lower gradient of the central quadrupole (QFM) between the two sextupoles (SF) at the dispersion peak helps to control second order vertical chromaticity. In addition, to control the increase of the third order horizontal chromaticity, a

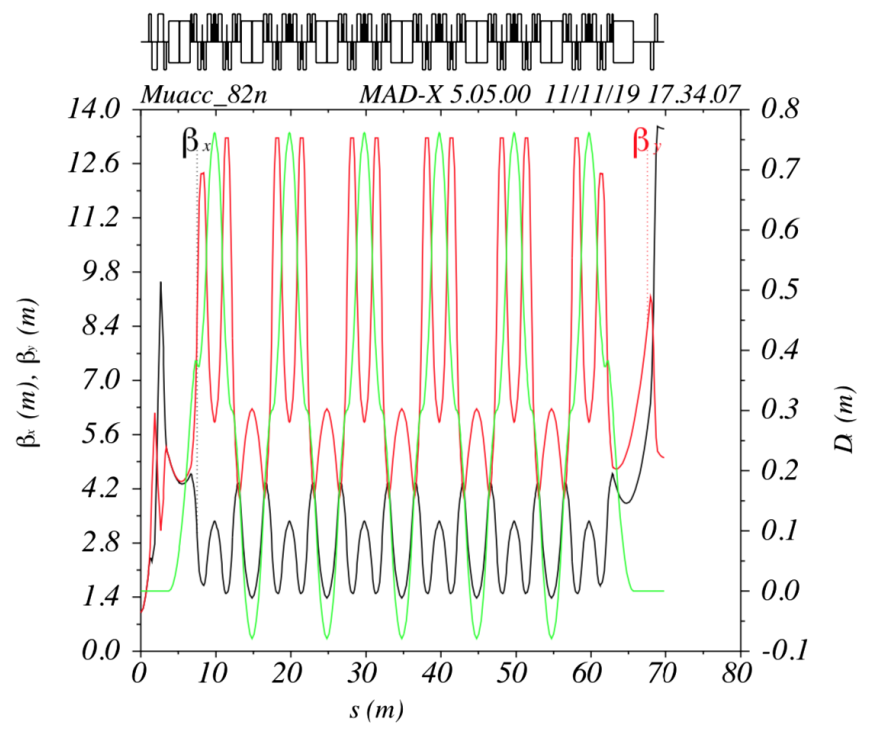

FIG. 2. Optical functions of half of the muon accumulator ring; from the center of the insertion region to the center of the $\mathrm{rf}$ section.

weak defocusing gradient has been added to each dipole of the four central arc cells.

Figure 4 shows the low- $\beta$ insertion region (IR) with the muon production target placed at the minimum $\beta$ location, similar to an interaction point (IP) in a collider. In the plot the IP is at $s=0 \mathrm{~m}$, where $\beta^{*}$ is $1 \mathrm{~m}$ in both planes $\mathrm{x}$ and $\mathrm{y}$; here the first and high order dispersion terms are canceled. The distance between the IP and the first quadrupole, $\ell^{*}$, is $1 \mathrm{~m}$.

Since this region is in common for the two $\mu^{+}$and $\mu^{-}$ beams the IR gives the same chromaticity contribution to both $\mathrm{x}$ and $\mathrm{y}$ planes. Positrons pass through the muon production target sharing the IR with $\mu^{+}$and $\mu^{-}$beams,

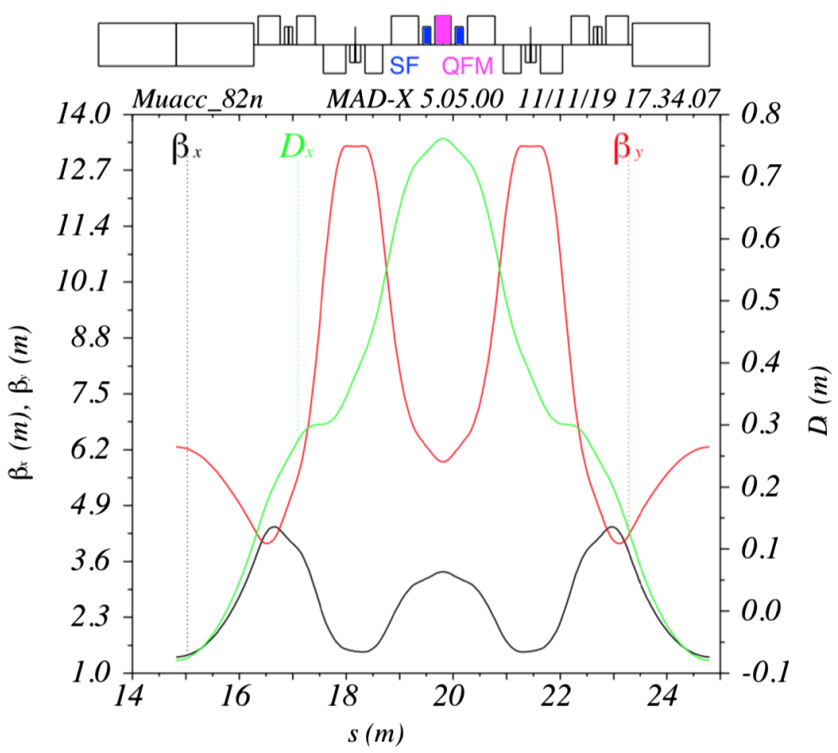

FIG. 3. A central cell of the muon accumulator ring. 


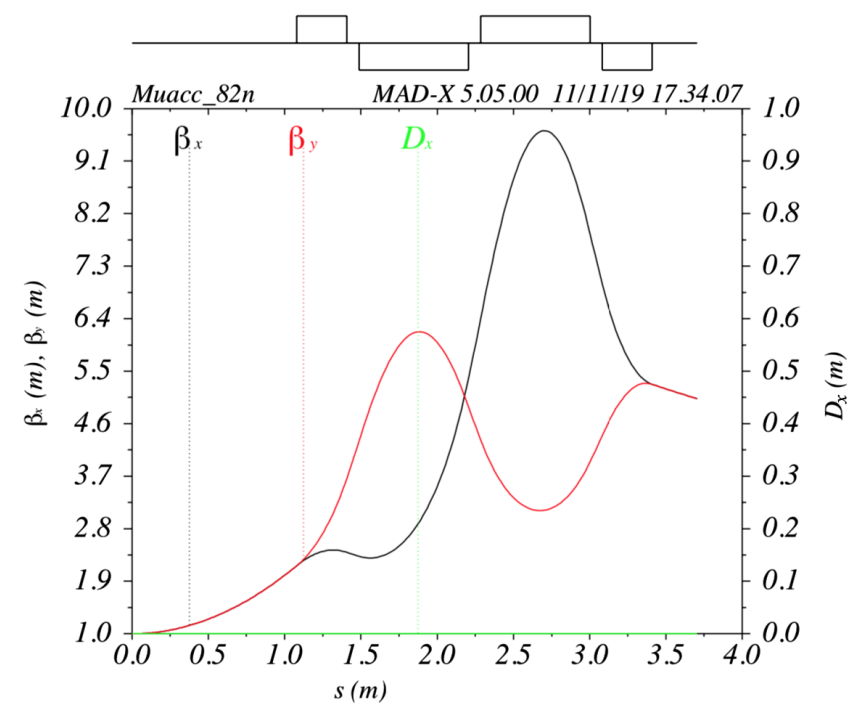

FIG. 4. Half section of the insertion region, the muon target is placed at $s=0 \mathrm{~m}$.

thanks to a septum and a bending magnet. The bending magnet is split in two, being the first piece in common with the three beams and its length and bending angle is optimized to achieve the maximum separation of the three beams. Since these magnets are included in the ring lattice, it is possible to perform an overall optimization of the energy acceptance, preventing particle losses in the subsequent extraction section.

This accumulator ring has a radio frequency cavity to recover the energy loss of the muon beam due to the passage through the target. A careful optimization of the nonlinear terms obtained a very small momentum compaction factor, as small as $\alpha_{c} \sim 7 \times 10^{-5}$, with a linear slope up to very high energy deviations. This allows very offmomentum muon particles to return to the same phase space location turn-by-turn. The rf straight section houses also two quadrupoles, enabling a small chromaticity, and large available drift space. The center of this rf section corresponds to large values of the horizontal beta function, providing for an easier extraction of the muon beam.

Chromaticity correction is an essential ingredient for the required large energy acceptance, needed up to high order terms. This is performed with a family of sextupoles placed in proper locations of small-beta and high-dispersion values. Second order momentum compaction cancellation is obtained with a family of sextupoles placed in proper locations where the beta function is large and dispersion is small. To correct chromaticity and high orders momentum compaction simultaneously five independent sextupoles strengths for each cell have been optimized according to the following conditions: minimum horizontal dimension at the target as a function of the muon beam energy, i.e., minimum variation of the beta function at the target as a function of energy, maximum energy bandwidth, minimum detuning as a function of energy, and maximum linearity of
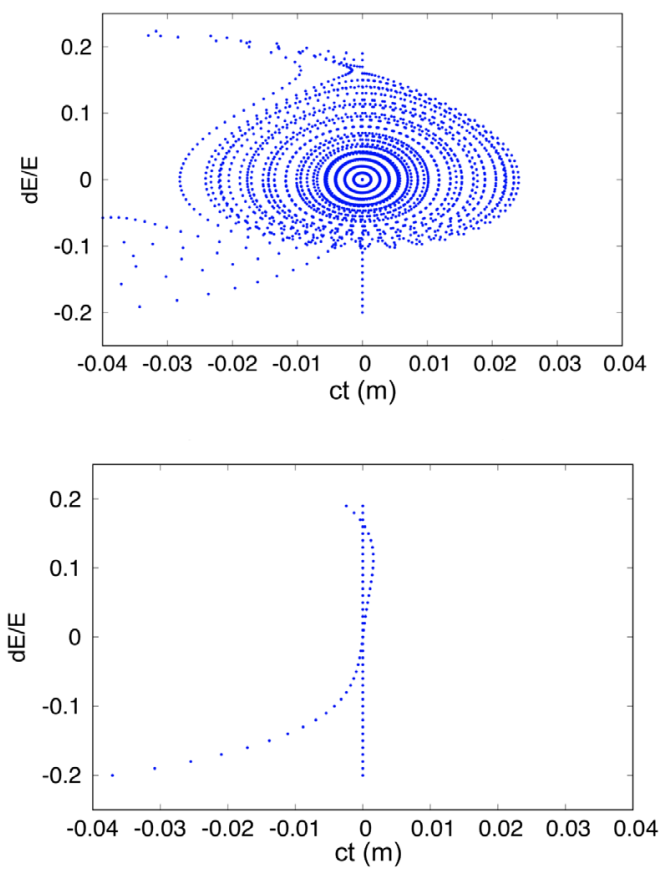

FIG. 5. Longitudinal phase space for the accumulator ring from MAD-X PTC for rf on (upper) and rf off (lower).

the momentum compaction. All the lengths of the elements have been optimized not only as a function of the dynamic aperture maximization but also considering the realistic magnetic fields and gradients obtainable with the $\mathrm{Nb}_{3} \mathrm{Ti}$ technology.

We performed particle tracking with this optics using MAD-X PTC and Accelerator Toolbox (AT) [10]. A good agreement has been found between MAD-X PTC and AT. Figure 5 shows the longitudinal phase space from MAD-X PTC with and without rf switched on, upper and lower plots, respectively. The upper plot shows an energy acceptance of $-10 \%,+15 \%$ for the bare ring, without target. The slope of the curve of the lower plot gives an

TABLE I. Parameters table of the muon accumulator ring.

\begin{tabular}{lcc}
\hline \hline Muon beam energy & $\mathrm{GeV}$ & 22.5 \\
Circumference & $\mathrm{m}$ & 140 \\
Number of cells & $\mathrm{GHz}$ & 12 \\
rf frequency & $\mathrm{MV}$ & 3.9 \\
rf voltage & & 200 \\
Harmonic number & & 1100 \\
Number of bunches & & 8.84 \\
Horizontal betatron tune & & 3.73 \\
Vertical betatron tune & & 0.015 \\
Longitudinal tune & & $-7.12 \times 10^{-5}$ \\
Momentum compaction & & -10.28 \\
Natural horizontal chromaticity & & 0.9 \\
Natural vertical chromaticity & $\mathrm{cm}$ & $-10 \%,+15 \%$ \\
Bunch length & & \\
Ring energy acceptance & & \\
\hline \hline
\end{tabular}


indication of the linearity of the momentum compaction factor.

The final ring parameters are summarized in Table I.

\section{MUON TRACKING SIMULATION AND TARGET OPTIONS}

The large energy acceptance optics described in the previous section has been used to perform particle tracking through the ring with different targets, allowing dedicated studies for the characterization of the accumulated muon beams. To perform these studies we have used the fast Monte Carlo simulation code, MUFASA. MUFASA includes the relevant processes that occur when either a positron or a muon beam traverses a target. It accounts for the muon production process $e^{+} e^{-} \rightarrow \mu^{+} \mu^{-}$, the multiple scattering, the ionization, and bremsstrahlung energy loss according to the formulas reported in Ref. [11]. Results have been compared with those obtained with GEANT4 [12], showing an excellent agreement.

In this study we use the statistical definition of the emittance

$$
\epsilon=\sigma_{x} \sigma_{x^{\prime}} \sqrt{1-r^{2}}
$$

where $\sigma_{x}$ and $\sigma_{x^{\prime}}$ are the beam size and divergence, respectively and $r$ is the correlation coefficient. The emittance is relevant to determine the final luminosity at the collider: $\mathcal{L} \propto N^{2} / \epsilon$; where $N$ is the number of muons per bunch. The number of $\mu^{+} \mu^{-}$pairs produced per positron bunch on target is given by:

$$
n\left(\mu^{+} \mu^{-}\right)=n^{+} \rho^{-} L \sigma\left(\mu^{+} \mu^{-}\right)
$$

where $n^{+}$is the number of positrons in the bunch, $\rho^{-}$is the electron density in the medium, $L$ is the thickness of the target, and $\sigma\left(\mu^{+} \mu^{-}\right)$is the muon pair production cross section. Since bremsstrahlung and multiple scattering are the dominant processes for the positron beam at these energies, the target thickness and material are the key parameters that play a role in the muon production optimization.

We refer to the muon production efficiency eff $\left(\mu^{+} \mu^{-}\right)$, which is defined as the ratio of the number of produced $\mu^{+} \mu^{-}$pairs to the number of the incoming positrons:

$$
\operatorname{eff}\left(\mu^{+} \mu^{-}\right)=\frac{n\left(\mu^{+} \mu^{-}\right)}{n^{+}} .
$$

Figure 6 shows the muon production efficiency obtained with MUFASA for a $45 \mathrm{GeV}$ positron beam hitting targets of different materials and thickness as a function of radiation lengths, $\mathrm{X}_{0}$. The results of simulations performed with targets based on carbon $(\mathrm{C})$, beryllium (Be), liquid lithium (LLi), and liquid hydrogen (LH) show a clear enhancement

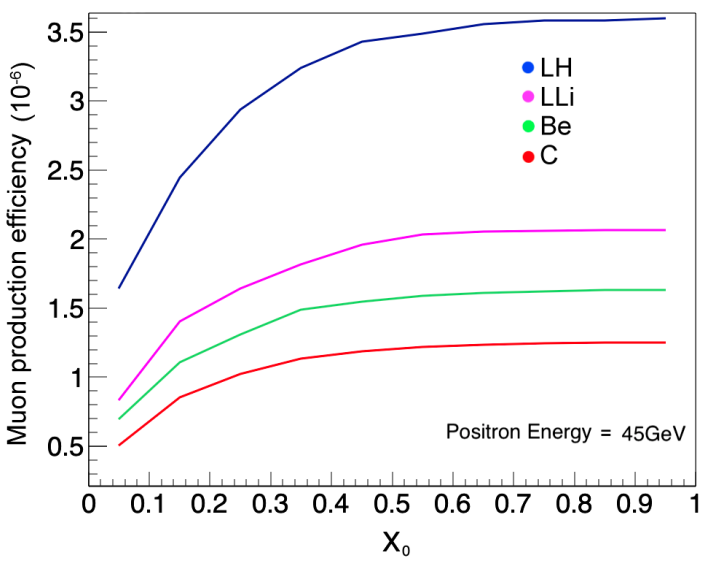

FIG. 6. Muon production efficiency for a $45 \mathrm{GeV}$ positron beam impinging on different materials as a function of $\mathrm{X}_{0}$ : carbon $(\mathrm{C})$, beryllium (Be), liquid lithium (LLi), and liquid hydrogen (LH).

of the production efficiency for lighter materials, in agreement with expectations.

We performed simulations varying the positron beam energy, the target thickness, and the target material to study the effect on muon beam parameters. A positron beam energy range [44-58] GeV with a positron emittance of $7 \times 10^{-10} \mathrm{~m}$ rad and transverse beam size of $20 \mu \mathrm{m}$ in both planes has been considered. The thickness of targets has been varied in the range $\mathrm{X}_{0}=[0.1-0.9]$, with corresponding lengths listed in Table II. Two materials have been considered for this study: beryllium, and liquid lithium.

Figure 7 shows the results of this study. The behavior of the beam size, beam divergence and the muon production efficiency is shown as a function of the positron beam energy for different target thickness. Results obtained for Be and LLi targets are given on the left and right of Fig. 7, respectively. The beam size is shown to be proportional to the target thickness, having lower values for Be targets. The muon beam divergence depends mainly on the positron beam energy, being dominated by the production kinematics. The small increase for thicker targets is due to the contribution of multiple scattering. The muon production efficiency behavior resembles the production cross section energy dependence.

The muon energy distribution at production is, in good approximation, uniform with maximum and minimum

TABLE II. Thickness in units of $\mathrm{X} 0$ for the two materials considered in simulations, Be and LLi.

\begin{tabular}{lcc}
\hline \hline Thickness & Be $[\mathrm{m}]$ & LLi $[\mathrm{m}]$ \\
\hline 0.1 X0 & 0.035 & 0.155 \\
0.3 X0 & 0.106 & 0.465 \\
0.5 X0 & 0.176 & 0.775 \\
0.7 X0 & 0.247 & 1.085 \\
0.9 X0 & 0.317 & 1.395 \\
\hline \hline
\end{tabular}




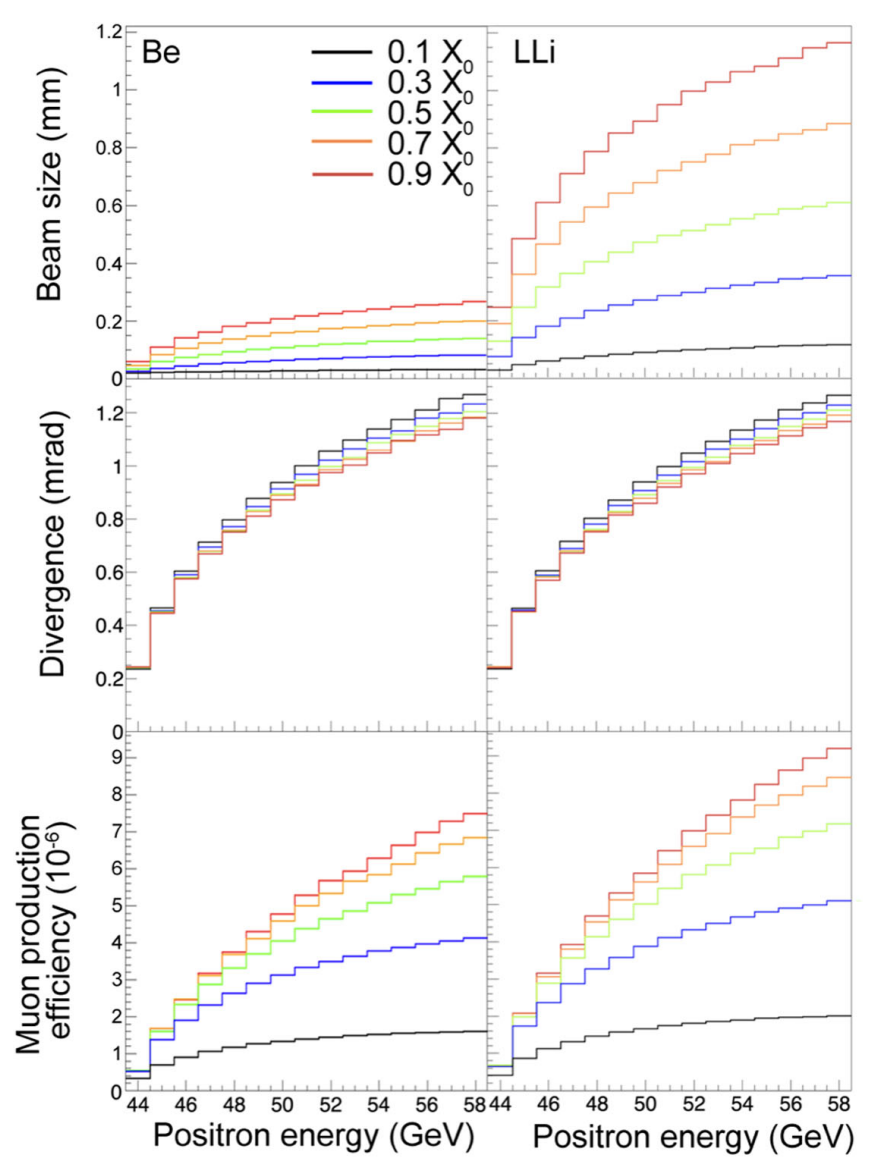

FIG. 7. Muon beam at production for liquid Li (right column) and Be (left column) targets. Plots from top to bottom: beam size, divergence, and number of produced muons.

values given by kinematics. The maximum, mean and minimum values of the muons energy are shown in Fig. 8. The muon energy distribution is independent of the target material and mildly dependent on the target thickness; only results for the Lithium target are shown in Fig. 8.

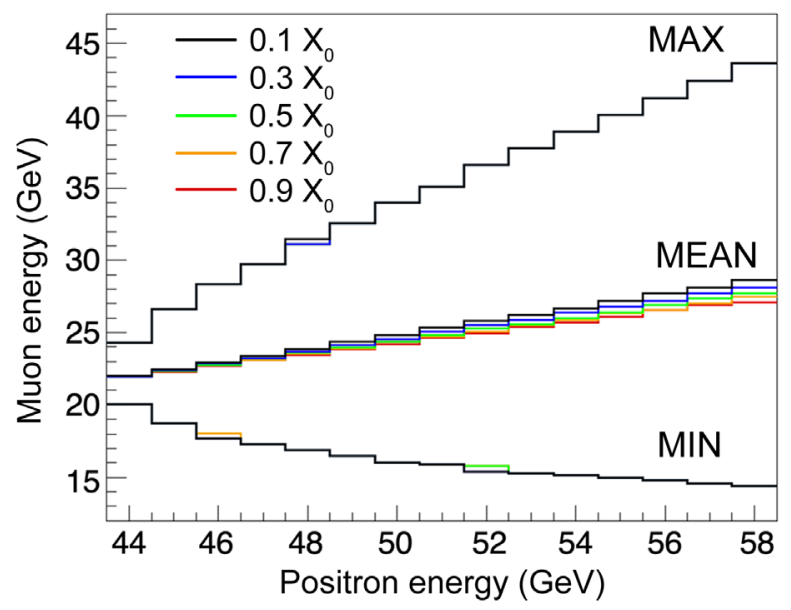

FIG. 8. Maximum, mean and minimum value of the muon beam energy at production as a function of the positron beam energy for different LLi target lengths.
In summary, Fig. 7 shows that an increase of the positron beam energy would enhance the production efficiency, provided that the energy acceptance of the muon collecting system matches the requirements shown in Fig. 8. In addition, it has been shown that Be targets permit smaller muon beam sizes and smaller emittances, thus better performances even though the muon production efficiency is slightly smaller with respect to LLi.

The liquid lithium and liquid compound targets have been subject of further studies in the form of film jets. These studies are discussed in III A and III B.

To assess the number of muons per bunch and the bunch emittance at the end of the accumulation process, we performed multiturn particle tracking studies using MUFASA. We first considered a setup with a beryllium target placed at the insertion region center of the accumulator ring described in Sec. II. We used a positron beam energy of $45 \mathrm{GeV}$ to match the energy acceptance of the lattice, and a target thickness of $0.3 \mathrm{X}_{0}$; thicker targets, for example $0.5 \mathrm{X}_{0}$, do not substantially improve $N^{2} / \epsilon$ while enhancing the scattering effect during the accumulation process. The positron beam at the target position has a transverse size of $20 \mu \mathrm{m}$ with an emittance of $7 \times 10^{-10} \mathrm{~m} \mathrm{rad}$. We considered up to 1500 bunches with $5 \times 10^{11} e^{+} /$bunch. Thermomechanical stresses related to this configuration are described in Ref. [3].

Figure 9 shows the number of accumulated muons per bunch as a function of turns. After 1500 turns of accumulation ( $\sim 1.5$ muon lifetimes), a value of about of $3.5 \times$ $10^{8} \mu$ /bunch is obtained. Results of simulations performed excluding multiple scattering effects, and considering only loss due to muon decay are shown for comparison.

The beam size and the divergence, shown in Fig. 10, increase with the number of turns as an effect of the multiple scattering (see also [8]). The increase is not observed when multiple scattering is not included in the simulations (dashed curves in Fig. 10). In this case the

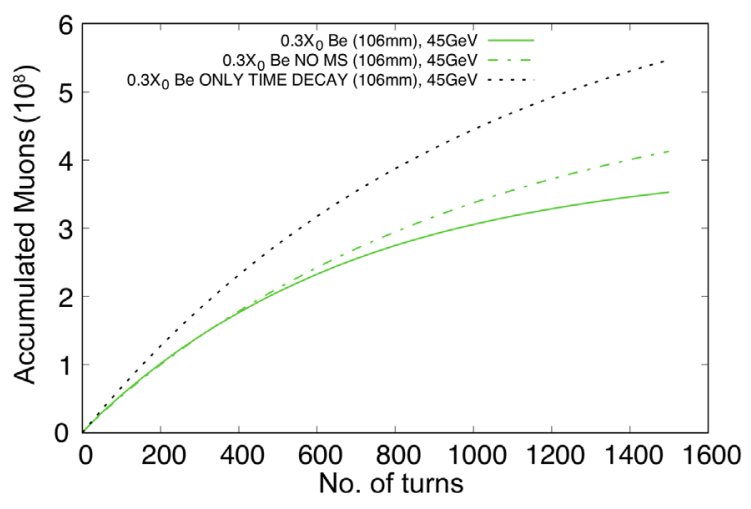

FIG. 9. Number of muons as a function of accumulation turns for a $45 \mathrm{GeV}$ positron beam impinging on a $0.3 \mathrm{X}_{0}$ thick Be target with (solid line) and without (dashed dot line) including multiple scattering. The ideal accumulation, losses due to muon decay only, is the black dotted line. 

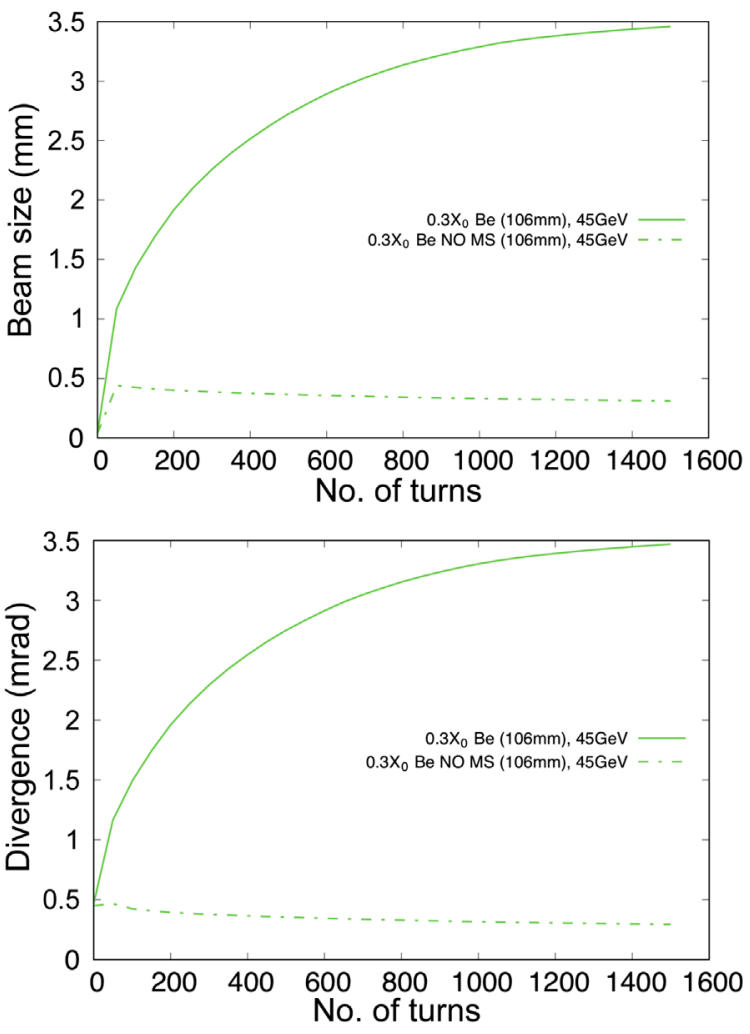

FIG. 10. Beam size and divergence as a function of accumulation turns for a $45 \mathrm{GeV}$ positron beam impinging on a $0.3 \mathrm{X}_{0}$ thick Be target with (solid line) and without (dashed line) including multiple scattering.

simulation shows ionization cooling effects due to the energy loss in target and the energy gain from radio frequency cavity. As shown in Fig. $11 N^{2} / \epsilon$ is degraded by multiple scattering contribution affecting muon beam emittance during accumulation. We note that the $\beta^{*}=1 \mathrm{~m}$ is not optimized for a Be target of $10.6 \mathrm{~cm}$, so the emittance increase could be reduced with a proper beam matching.

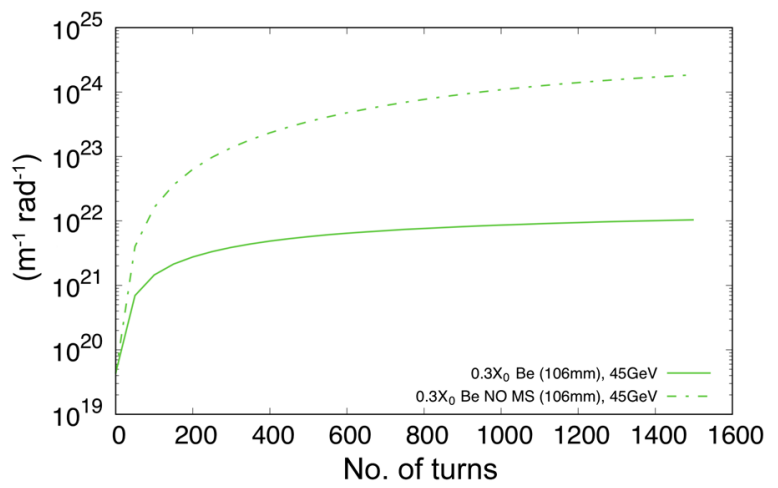

FIG. 11. $N^{2} / \epsilon$ as a function of accumulation turns for a $45 \mathrm{GeV}$ positron beam impinging on a $0.3 \mathrm{X}_{0}$ thick Be target with (solid line) and without (dashed line) including multiple scattering.

\section{A. Film jet target}

We discuss in this section the behavior of the muon beam using a film jet target, with the goal of providing a reduction of the multiple scattering effect. By film jet target we define a liquid target with transverse dimension comparable to the muon beam size $[\mathcal{O}(50 \mu \mathrm{m})]$. As the muon beam size exceeds the target transverse dimension, a fraction of the muon beam no longer experiences the target perturbation. We considered a film jet liquid lithium target of $0.3 \mathrm{X}_{0}$, corresponding to $46.5 \mathrm{~cm}$. Liquid lithium is under study for diverters in tokamaks and in use for neutron production [13]. Liquid hydrogen has shown high evaporations rates hardly sustainable with present technology.

We studied the muon bunch parameters using $45 \mathrm{GeV}$ positron beam (up to 1500 bunches with $5 \times 10^{11} e^{+} /$ bunch) with a transverse beam size of $20 \mu \mathrm{m}$ and an emittance of $7 \times 10^{-10} \mathrm{~m} \mathrm{rad}$. A film-jet target is placed at the insertion region center with $\beta^{*}=1 \mathrm{~m}$.

Figure 12 shows the number of accumulated muons as a function of machine turns, for targets of different transverse dimensions and also for a target where multiple scattering is not included in the simulations. The number of muon losses decrease as the target transverse dimension decreases and approaches the ideal case, no multiple scattering, for target widths of $50 \mu \mathrm{m}$.

Figure 13 shows the behavior of the beam size and the divergence of the accumulated muon beam. As expected, the effect of multiple scattering is strongly reduced for thinner targets. The slight decrease of beam size and divergence observed in the case where multiple scattering is not included in the simulations is due to the cooling provided by ionization energy loss.

Figure 14 shows $N^{2} / \epsilon$ as a function of the accumulator turns for the same lithium targets discussed above. For the $50 \mu \mathrm{m}$ wide lithium target, a value of $\sim 3.3 \times 10^{23}$ is

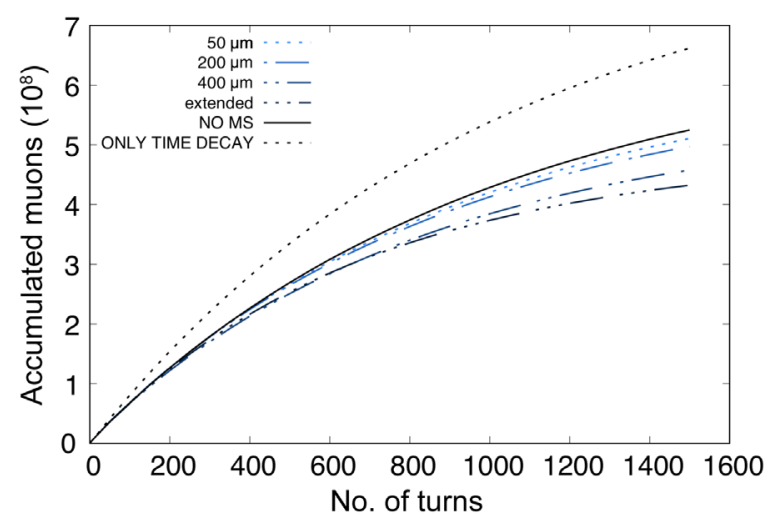

FIG. 12. Number of muons as a function of accumulation turns for a $45 \mathrm{GeV}$ positron beam impinging on a $0.3 \mathrm{X}_{0} \mathrm{LLi}$ target for four different widths (dashed lines), compared with the extended target with multiple scattering switched off (solid line). The ideal accumulation, losses due to muon decay only, is the black dotted line. 

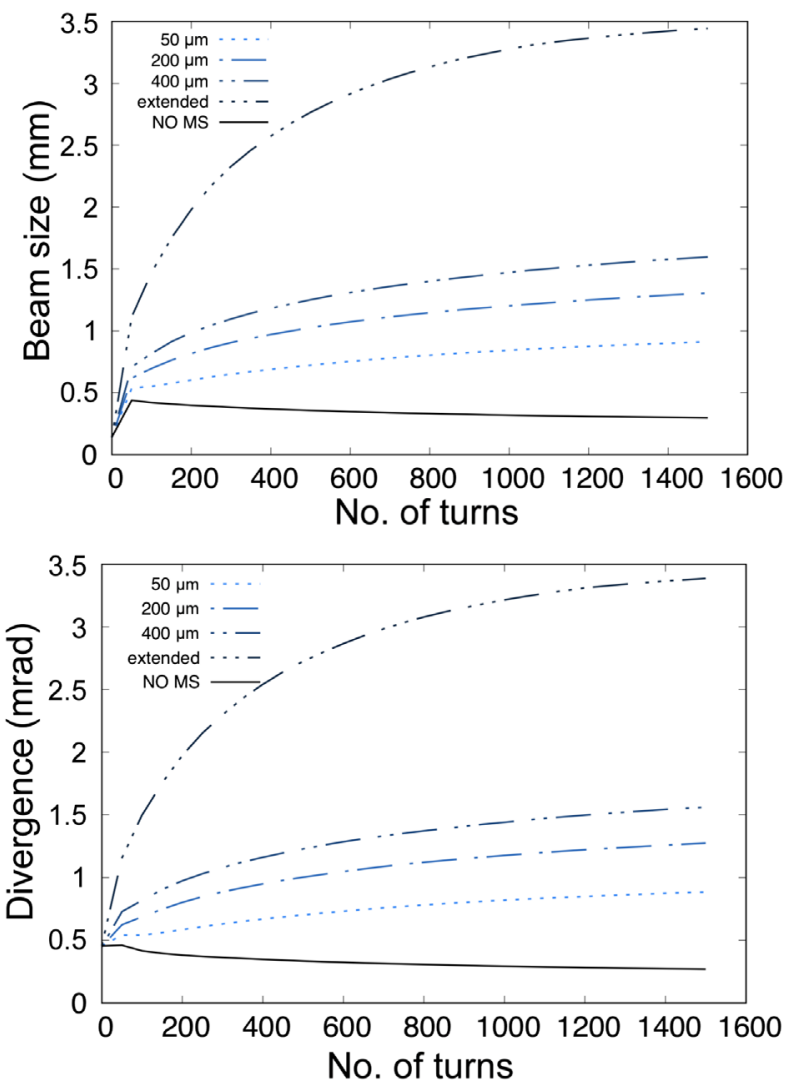

FIG. 13. Beam size and divergence as a function of accumulation turns for a $45 \mathrm{GeV}$ positron beam impinging on a $0.3 \mathrm{X}_{0}$ LLi target for four different widths (dashed lines), compared with the extended target with multiple scattering switched off (solid line).

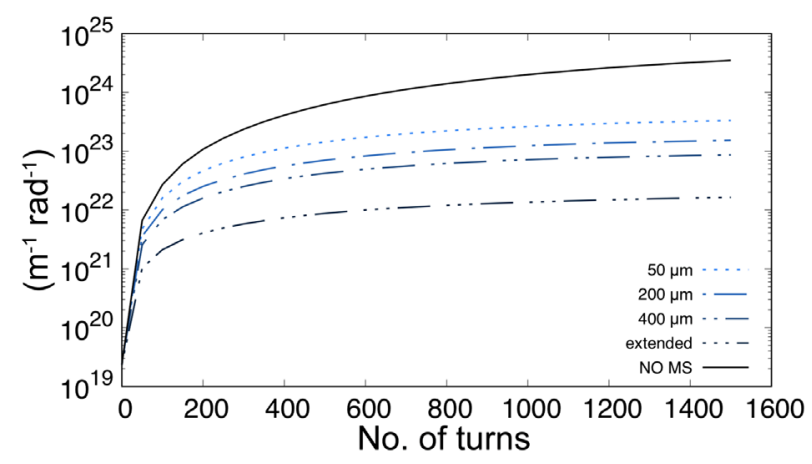

FIG. 14. $\quad N^{2} / \epsilon$ as a function of accumulation turns for a $45 \mathrm{GeV}$ positron beam impinging on a $0.3 \mathrm{X}_{0}$ thick LLi target for four different widths (dashed lines), compared with the extended target with multiple scattering switched off (solid line).

obtained for $N^{2} / \epsilon$ after 1500 machine turns; a factor $\sim 30$ higher than that obtained with the beryllium target.

\section{B. Liquid lithium-diamond target}

In the previous section we have shown that using a liquid lithium film jet target yields an improvement in the quality of the accumulated muon beam, as it mitigates the effect of multiple scattering. However liquid targets have in general low density, liquid Lithium has a radiation length of $155 \mathrm{~cm}$, inducing an increase of the beam size.

A possible solution to reduce the length of the target is to mix liquid lithium (LLi) with diamond (D) powder. The average density and radiation length of the mixed material target is given by:

$$
\frac{\rho}{\mathrm{X}_{0}}=f(\mathrm{LLi}) \frac{\rho \mathrm{LLi}}{\mathrm{X}_{0}^{\mathrm{LLi}}}+f(\mathrm{D}) \frac{\rho^{\mathrm{D}}}{\mathrm{X}_{0}^{\mathrm{D}}}
$$

where $f$ is the fraction of the target length occupied by a given material, $\rho$ is the density in $\left[\mathrm{g} \mathrm{cm}^{-3}\right]$ and $\mathrm{X}_{0}$ is the radiation length in $\left[\mathrm{g} \mathrm{cm}^{-2}\right]$. The values of these quantities for different compositions are given in Table III. From Table III we can see that using a compound made of $10 \%$ of liquid lithium and $90 \%$ of diamond powder (10\%LLi$90 \% \mathrm{D}$ ), the radiation length can be reduced by more than a factor of ten with respect to the pure liquid Lithium, while keeping the liquid state and thus the possibility to make a film jet target. On the other hand, due to the fact that the target is now mostly diamond, the muon production efficiency will be slightly reduced as shown previously in Fig. 6. To effectively reduce the beam size the value of the $\beta$ function at the target insertion has to be matched with the target length. Starting from the lattice described in Sec. II, the value of the $\beta$ at the interaction point has been reduced in order to study the impact on the muon beam quality. Since chromaticity was not re-optimized, the tracking was performed considering an on-energy muon beam. For this study, a $0.5 \mathrm{X}_{0}(6.7 \mathrm{~cm})$ thick $10 \% \mathrm{LLi}-90 \%$ D film jet target $50 \mu \mathrm{m}$ wide has been used. Different fractions down to $50 \%$ of LLi and 50\% diamond powder with same $\mathrm{X}_{0}$ gave similar results. The behavior of the beam size and divergence of the accumulated muon beam are shown in Fig. 15. As expected, the beam size and the divergence are respectively directly and inversely proportional to $\beta^{*}$. The final emittance of the accumulated muon beam is directly proportional to the $\beta^{*}$, as shown in Fig. 16. For a lattice with $\beta^{*}=0.1 \mathrm{~m}$ the emittance is $3.33 \times 10^{-7} \mathrm{~m} \mathrm{rad}$, about a factor two smaller with respect to the $\beta^{*}=1 \mathrm{~m}$ case.

TABLE III. Properties of LLi-D compounds, for different fractions of liquid lithium $f(\mathrm{LLi})$, and diamond powder $f(\mathrm{D})$.

\begin{tabular}{lcccr}
\hline \hline$f(\mathrm{LLi})$ & $f(\mathrm{D})$ & $\rho\left[\mathrm{g} \mathrm{cm}^{-3}\right]$ & $\mathrm{X}_{0}\left[\mathrm{~g} \mathrm{~cm}^{-2}\right]$ & $\mathrm{X}_{0}[\mathrm{~cm}]$ \\
\hline 1.0 & 0.0 & 0.534 & 82.78 & 155.02 \\
0.9 & 0.1 & 0.833 & 59.26 & 71.18 \\
0.7 & 0.3 & 1.430 & 48.89 & 34.19 \\
0.5 & 0.5 & 2.027 & 45.61 & 22.50 \\
0.3 & 0.7 & 2.624 & 44.00 & 16.77 \\
0.1 & 0.9 & 3.221 & 43.04 & 13.36 \\
0.0 & 1.0 & 3.520 & 42.70 & 12.13 \\
\hline \hline
\end{tabular}



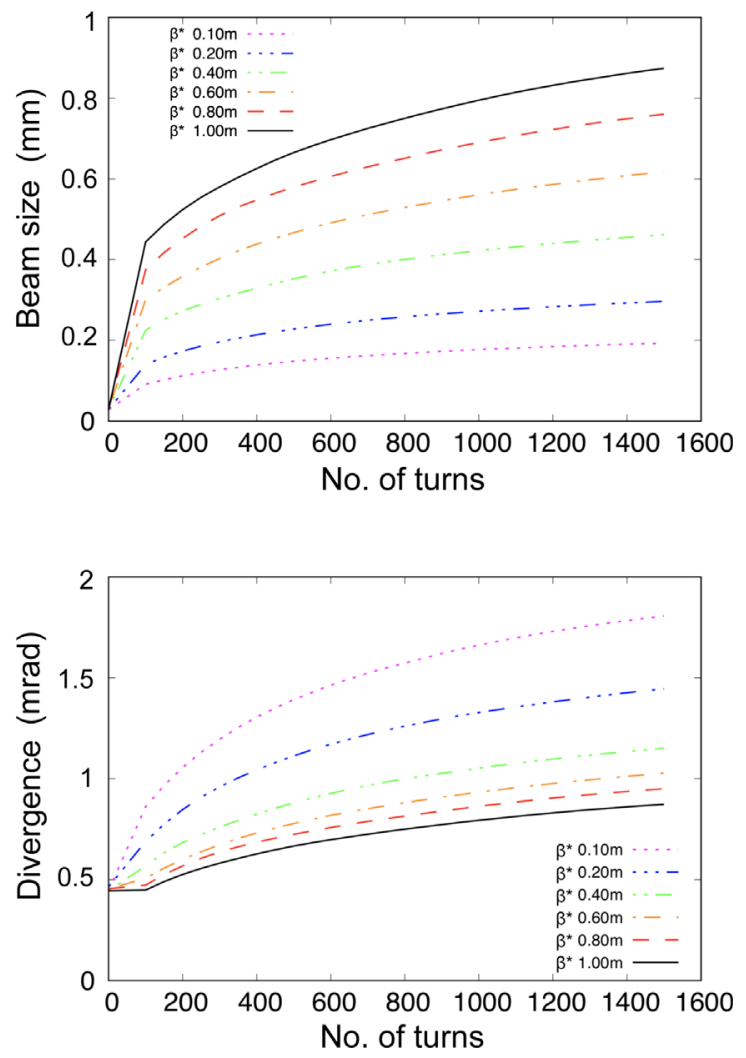

FIG. 15. Beam size and divergence as a function accumulation turns for a $45 \mathrm{GeV}$ positron beam impinging on a $10 \% \mathrm{LLi}-90 \%$ diamond powder target, $0.5 \mathrm{X}_{0}$ thick and $50 \mu \mathrm{m}$ wide, for different values of $\beta^{*}$.

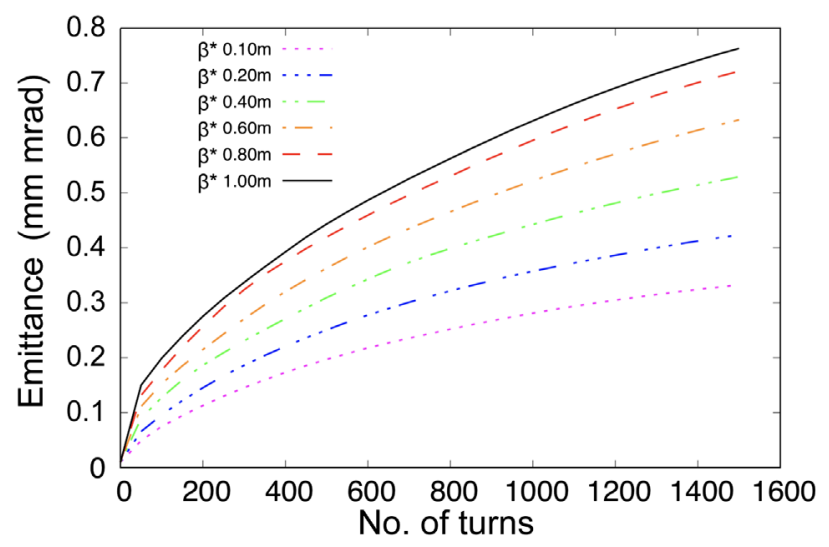

FIG. 16. Muon beam emittance as a function of accumulation turns for a $45 \mathrm{GeV}$ positron beam impinging on a $10 \% \mathrm{LLi}-90 \%$ diamond powder target, $0.5 \mathrm{X}_{0}$ thick and $50 \mu \mathrm{m}$ wide, for different values of $\beta^{*}$.

\section{The revolver configuration}

The muon bunch parameters can be further improved by a different injection configuration of the positron beam hitting the target. The concept is to profit from the small positron beam emittance and simultaneously inject multiple
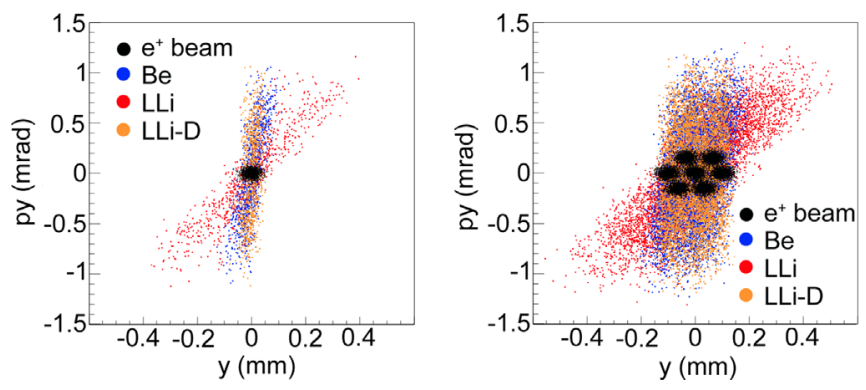

FIG. 17. Vertical phase space of the positron beam at target (in black), over-imposed is the muon beam produced by three different targets $0.3 \mathrm{X}_{0}$ thick: Be (blue dots), LLi (red dots), LLi-D (orange dots). Left and right plots show the standard single bunch and the revolver configuration, respectively.

bunches with proper vertical spacing (y) and kick (y' and/or $\left.x^{\prime}\right)$. This scheme can be implemented with appropriate delay lines and kickers as sketched in Fig. 1. This configuration provides a reduction in the number of turns needed, thus implying higher muon survival probability. In addition a smaller multiple scattering effect is present thanks to the smaller radiation length experienced by muons. A possible scheme is illustrated in Fig. 17 where the vertical phase space is shown for the standard positron beam (left plot) and for a revolver configuration in the case of seven bunches (right plot). The seven $e^{+}$bunches are simultaneously injected with vertical off-set y and kick y'. The muons produced by these bunches hitting a LLi-D film jet have been tracked with MUFASA through the ring and compared to the single $e^{+}$bunch case. Figure 18 shows that the muon beam size and divergence is not affected by this configuration of the positron beam at target. The great benefit of the revolver configuration is the increase in the number of muons per bunch with a net improvement in the horizontal and vertical emittance, as the number of required turns is decreased (see upper plot in Fig. 19). This

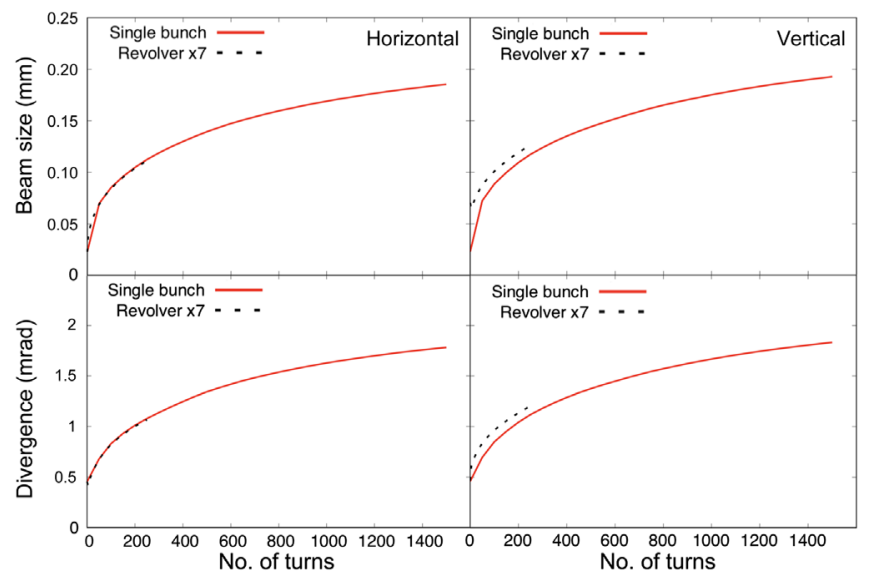

FIG. 18. Muon bunch size and divergence for the revolver configuration (black dashed curve) compared to the standard single bunch case (red line) for a LLi-diamond powder target. 

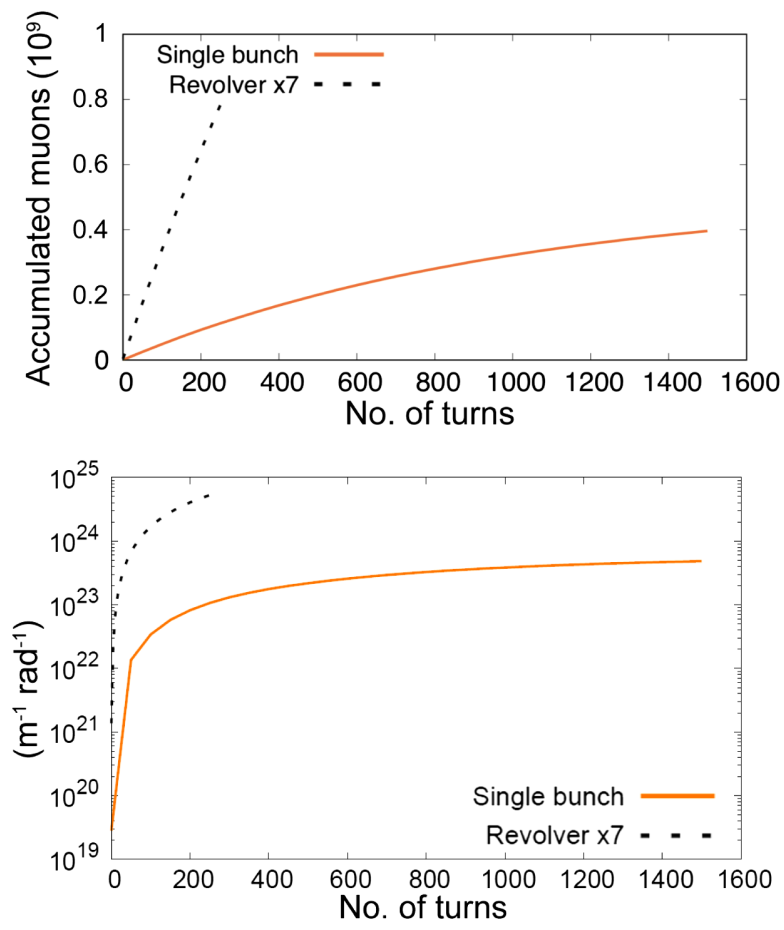

FIG. 19. Muon accumulation (upper) and $N^{2} / \epsilon$ (lower) for the revolver configuration with seven bunches indicated by a dashed line and the standard single bunch case indicated by a plain line. Results refer to: $45 \mathrm{GeV}$ positron beam, $1500 e^{+}$bunches with $5 \times 10^{11} e^{+} /$bunch, $0.3 \mathrm{X}_{0}$ LLi-diamond target.

improvement translates in a gain of about two orders of magnitude in $N^{2} / \epsilon$, as appears from the lower plot in Figure 19.

\section{CONCLUSION}

We have presented start-to-end simulations of production and accumulation of muon beams produced by positrons on target. Muon accumulator rings have been designed and their optics described. These rings are compact, about $140 \mathrm{~m}$, with a very large energy acceptance, $-10 \%,+15 \%$, and a proper low- $\beta$ section to host the target insertion. Such a novel optics has possible interesting applications in future circular colliders as well as to last generation synchrotron light sources. The former at very high energy are dominated by beamstrahlung, while the latter are limited by the Touschek lifetime due to the small emittances.

The study has been performed for various targets considering different parameters. We have shown how the suppression of multiple scattering needs to be balanced with the target length, which brings an intrinsic emittance increase and foresees the use of a film target. A possible solution is represented by a film target made by a compound of diamond power and liquid lithium. We have presented results for a $50 \mu \mathrm{m}$ LLi-D film jet of about $0.5 \mathrm{X}_{0}$ corresponding to $6.7 \mathrm{~cm}$ using $\beta^{*}$ as low as $0.1 \mathrm{~m}$. An emittance for the $\sim 22 \mathrm{GeV}$ muon beam of about
$0.3 \times 10^{-6} \mathrm{~m}$ rad has been obtained after about one thousand accumulation turns. Further improvements can be obtained reducing the $\beta^{*}$.

We have also shown that the number of turns in the accumulator can be decreased with the so called revolver configuration resulting in an increase of the muons survival with smaller muon bunch perturbation, i.e., smaller emittance. In this case an emittance of about $0.1 \times 10^{-6} \mathrm{~m} \mathrm{rad}$ and a number of muons per bunch of about $10^{9}$ have been obtained. We have also shown that an increase in the energy acceptance would allow operation at higher positron energy, thus gaining in production efficiency. Such an increase requires improvement in the chromaticity correction in the present optics or/and alternative solutions as the use of FFA lattice that provides energy acceptance close to 20\% [14].

Finally, the possibility of synchrotron radiation damping at high energy, i.e., FCC [15] tunnel, is an additional effect that plays a role to reduce the final emittance and will be subject of further studies.

\section{ACKNOWLEDGMENTS}

We thank the late Lewis Keller. Lewis has been one of the main actors of the LEMMA proposal. In particular he has pioneered the study on the accumulation effects, deeply investigated in this paper. The authors thank O. R. BlancoGarcía for the set-up of the MAD-X PTC interface, N. Carmignani for useful discussions, and the LEMMA team.

[1] M. Antonelli, M. Boscolo, R. Di Nardo, and P. Raimondi, Novel proposal for a low emittance muon beam using positron beam on target, Nucl. Instrum. Methods Phys. Res., Sect. A 807, 101 (2016).

[2] M. Boscolo, M. Antonelli, O. R. Blanco-Garcia, S. Guiducci, S. Liuzzo, P. Raimondi, and F. Collamati, Low emittance muon accelerator studies with production from positrons on target, Phys. Rev. Accel. Beams 21, 061005 (2018).

[3] D. Alesini et al., Positron driven muon source for a muon collider, arXiv:1905.05747.

[4] M. Boscolo, J. P. Delahaye, and M. Palmer, The future prospects of muon colliders and neutrino factories, Rev. Accel. Sci. Techol. 10, 189 (2019).

[5] C. Ankenbrandt, S. A. Bogacz, A. Bross, S. Geer, C. Johnstone, D. Neuffer, and M. Popovic, Low-energy neutrino factory design, Phys. Rev. Accel. Beams 12, 070101 (2009).

[6] G. Flanagan, R. Abrams, C. Ankenbrandt, M. Cummings, R. Johnson, M. Popovic, and A. Moreti, Using Project X as a proton driver for muon colliders and neutrino factories, Conf. Proc. C 100523, 4452 (2010).

[7] R. B. Palmer, Muon colliders, Rev. Accel. Sci. Techol. 07, 137 (2014).

[8] M. Boscolo et al., Muon accumulator ring requirements for a low emittance muon collider from positrons on target, in Proceedings, 9th International Particle Accelerator 
Conference (IPAC 2018): Vancouver, BC, Canada 2018, IPAC18 (2018), http://jacow.org/ipac2018/papers/ mopmf087.pdf.

[9] MAD-X, Methodical Accelerator Design. http://mad.web .cern.ch/mad.

[10] B. Nash, N. Carmignani, L. Farvacque, S. M. Liuzzo, T. Perron, P. Raimondi, R. Versteegen, and S. White, New functionality for beam dynamics in accelerator toolbox (at), in Proceedings, 6th International Particle Accelerator Conference (IPAC 2015): Richmond, VA, USA, 2015, IPAC15, 05 (2015), http://jacow.org/ipac2015/papers/ mopwa014.pdf.

[11] M. Tanabashi et al. (Particle Data Group), Review of particle physics, Phys. Rev. D 98, 030001 (2018).

[12] GEANT4, toolkit for the simulation of the passage of particles through matter, http://geant4.cern.ch.
[13] E. Wakai et al., Engineering validation for lithium target facility of the IFMIF under IFMIF/EVEDA project, Nucl. Mat. Energy 9, 278 (2016).

[14] J. B. Lagrange, R. B. Appleby, J. M. Garland, J. Pasternak, and S. Tygier, Racetrack FFAG muon decay ring for nuSTORM with triplet focusing, J. Instrum. 13, P09013 (2018).

[15] A. Abada et al. (FCC Collaboration), FCC-ee: The lepton collider: Future Circular Collider conceptual design report volume 2, Eur. Phys. J. Spec. Top. 228, 261 (2019); https:// fcc.web.cern.ch/.

Correction: The previously published Figure 7 had incorrect labels on the vertical axis of the bottom panels and has been replaced. 